\title{
Transcriptome analysis of four Poplars exposed to continuous salinity
}

\section{stress}

\begin{abstract}
Populus pruinosa and P. euphratica, are two desert poplars characterized by extreme adaptation to salt stress. In contrast, $P$. trichocarpa and $P$. tomentosa are two salt sensitive congeners. In order to identify the adaptations to salt stress that are common to each type of these poplars, we compared transcriptome changes in the four poplar species after exposure to continuous salt stress. We identified 7822 1:1 orthologs between the four species and 702 differentially expressed genes (DEGs) during the $48 \mathrm{~h}$ time course. The result of hierarchical clustering suggested that the patterns of gene expression were significantly different between the two types of poplar. These genes were mainly involved in 'Oxidoreductase activity', 'Primary metabolic processes', 'Transport' and 'Nucleic acid binding transcription factor activity'. Among these DEGs, in which the functions related to responses to salt exposure are significantly overrepresented, 11 genes were found to be continuously up or down-regulated in response to salt stress in the two types. We also found that most of the orthologous candidate genes evolved under purifying selection and one ortholog (GDSL) showed positive selection. These genes probably played important roles when their ancestral clade underwent adaptive divergence into the two types, differentiated by habitat salt-content. Keywords: Desert poplars; Transcriptome; Continuous salt stress; Differentially expressed genes; Expression patterns
\end{abstract}

\section{Introduction}

Salt stress is one of the most significant environmental factors that limit plant growth, development and productivity in semiarid and arid areas (Rengasamy, 2006). It is estimated that over $6 \%$ of the world's total land area and nearly $20 \%$ of irrigated land are adversely affected by salt stress, which consequently limits the yield of crops and even the geographical distribution of plants (Yeo, 1998; $\underline{\text { Tuteja, 2007; Munns and Tester, }}$ 2008). A high level of salt stress is generally considered to result in a series of negative effects on plants, including ion toxicity, osmotic stress, oxidative damage and even nutrient imbalance (Zhang and Shi, 2013; Cabot et al., 2014). The adverse effects of salt stress influence almost all growth stages and physiological processes in plants, including photosynthesis, protein synthesis, energy and lipid metabolism (Bazihizina et 
al., 2012; Álvarez and Sánchez - Blanco, 2014). The plants that have evolved tolerance mechanisms from the molecular to cellular and biochemical to physiological levels have adapted to survive in high salinity environments (Sahu and Shaw, 2009; Tang et al., 2015; Sun et al., 2016). However, any adaptive process is preceded by a response to the changing environment, and one such early-stage adaptive response is a change in dynamic transcriptomes, the products that are responsible for enabling organisms to achieve cellular and organismal homeostasis through the co-ordination of various molecular events (Gharat et al., 2016). Consequently, among the responses, changes in gene expression play the most important role, and up- or down-regulation of gene expression in one species compared to another can result in phenotypic differences in stress tolerance (Jiang et al., 2013). However, it remains unclear which genes play important roles in plant salt tolerance; the identification of such genes could allow a comprehensive understanding of the genetic mechanisms that control responses to environmental stress and a basis for effective engineering strategies to enhance salt stress tolerance in crops (Cushman and Bohnert, 2000; Witzel et al., 2009).

The availability of genome-wide expression profiles of mRNA is accelerating progress in identifying the genes that are differentially expressed under both control and high salinity stress conditions. In recent years, considerable attention has been directed toward elucidating the molecular basis of plant salt tolerance by comparative transcriptome analyses of halophytes or non-halophytes using microarrays and/or RNASeq technologies (Deyholos, 2010); targets have included Arabidopsis (Taji et al., 2004; Gong et al., 2005; Wong et al., 2006), poplar (Cohen et al., 2010; Beritognolo et al., 2011; Qiu et al., 2011), barley (Liu et al., 2016). However, between different species, particularly between sensitive and non-sensitive pairs of related species, overall changes in gene expression and physiological responses to salt stress vary greatly (Ingram and Bartels, 1996; Zhu, 2002; Shinozaki et al., 2003). Little is known about the extent to which gene expression and transcriptome dynamics under continued salinity stress differ between closely related species and salt-sensitive and salt-tolerant species.

The two sister poplar species P. pruinosa and P. euphratica both grow in deserts and are adapted to salt stress, although their degree of adaptation differs (Zhang et al., 2013; Zhang et al., 2014a). It is not known whether differences in gene expression during the process of becoming adapted to salt stress occurred in the common ancestor of the two species, or whether each species underwent separate adaptive changes. Examination of 
gene expression at the whole-transcriptome level is a powerful tool with which to characterize candidate genes involved in specific stresses and assign them to functional categories ( $\underline{O}$ 'Neil et al., 2014). In the current study, we compared transcriptome changes in the two desert poplars and in two salt-sensitive poplar species $(P$. trichocarpa and $P$. tomentosa) in response to continuous salinity stress. Differences in the dynamic expression profiles of these species under salt stress may provide useful insights into adaptive patterns of gene expression in the ancestral clade of the desert poplars (Lasky et al., 2014). Gene ontology (GO) enrichment analysis of co-expression genes revealed changes in several biological processes and metabolic pathways in response to continuous salinity stress. In addition, genes that are found to be continuously up or down-regulated in response to salinity stress in the salt-tolerant species or the salt-sensitive species may facilitate the identification of potentially suitable targets for biotechnological manipulation, with the aim of improving salt tolerance in the genus.

\section{Materials and methods}

\subsection{Plant materials and salinity stress}

In order to examine adaptive divergence in gene expression between the two desert poplars and the two salt-sensitive poplars, we examined transcriptome data for $P$. pruinosa, P. euphratica and P. tomentosa (Ma et al., 2013) from NCBI accessions SRP033639, SRP028829 and SRP028830, respectively. We also cultured calli induced from the shoots of $P$. trichocarpa, which included the control (without $\mathrm{NaCl}$ ) and saltstressed calluses ( $200 \mathrm{mM} \mathrm{NaCl}$ for $6,12,24$, and $48 \mathrm{~h}$ ), as previously described (Zhang et al., 2014b).

\subsection{RNA extraction, cDNA library preparation and sequencing}

The total RNA samples were isolated from the control (without $\mathrm{NaCl}$ ) and salt-stressed calluses ( $200 \mathrm{mM} \mathrm{NaCl}$ for $6,12,24$, and $48 \mathrm{~h}$ ) using the CTAB procedure (Chang et al., 1993). After RNA extraction and quality determination (Zhang et al., 2014b), we constructed five cDNA libraries with 200 base pair (bp) inserts from salt-stressed calli and controls. The libraries were sequenced using an Illumina (San Diego, CA, USA) Genome Analyzer platform according to the manufacturer's protocols, with a 100bp read length. Image output data from the sequencer were transformed into raw sequence data by base-calling. As before (Ma et al., 2013; Zhang et al., 2014b), three biological replicates were examined.

2.3. Filtration of reads and transcriptome assembly 
Raw reads were initially processed to obtain clean reads by removing adaptor sequences, empty reads, low quality sequences (i.e. those with large numbers of Ns), and reads with more than $10 \% \mathrm{Q}<20$ bases. The Illumina sequencing data sets are available in the NCBI Sequence Read Archive (SRA) database with the project accession number: PRJNA284202. In order to examine temporal profiles of gene expression and differences in expression between the two salt-tolerant and the two salt-sensitive poplar species under salt stress, we first obtained the protein coding sequences of $P$. pruinosa (unpublished data), P. euphratica (Ma et al., 2013), P. trichocarpa (Tuskan et al., 2006) and P. tomentosa (Ma et al., 2013). The coding sequences of P. euphratica, $P$. trichocarpa and P. pruinosa were identified using the genome sequence pipeline, whilst the coding sequences of $P$. tomentosa were predicted by the TransDecoder module in the Trinity software package from the contigs and assembled by Trinity using the default parameters. For genes with alternative splicing variants, the longest transcripts were selected, and transposable element-like genes and sequences with internal stop codons, incompatible reading frames, or a coding sequence length of $<150 \mathrm{bp}$ were removed. In this way, we obtained protein coding genes for $P$. pruinosa, $P$. euphratica, $P$. trichocarpa and P. tomentosa. We then used Inparanoid (Östlund et al., 2010), which is based on a strictly reciprocal blast rule, to identify orthologous genes between every pair of species. Multiparanoid (Alexeyenko et al., 2006) was applied to identify the orthologous genes from the four poplar species. Finally, we identified 1:1 orthologs between the four poplar species. The orthologous sequences were aligned using TBA (Threaded Blockset Aligner) (Blanchette et al., 2004) and perfectly aligned consensus blocks larger than $150 \mathrm{bp}$ were extracted.

\subsection{Assessment of differential gene expression}

We mapped the paired-end RNA-seq reads from each of the four poplar species onto its own predicted protein coding genes using the Bowtie2 (version 2.1.0) (Langmead et al., 2009) software package, with default parameters, to determine the level of gene expression. It should be noted that we did not map RNA-seq reads from all four species onto the $P$. trichocarpa genome, because the intrinsic divergence between the species could have resulted in poor mapping (Ma et al., 2013). Alignment output files from Bowtie were parsed using custom PERL scripts to calculate the number of reads aligned to the consensus blocks for each gene. Based on the final read assignments, we calculated standard RPKM values (numbers of reads per kilobase per million mapped reads on exon regions within a given gene; (Mortazavi et al., 2008)) for each coding 
gene. We selected only genes that had RPKM $\geq 1$ in samples from two or more time points for further analysis. To compare expression levels of the selected genes across all time points, we applied the upper quartile normalization procedure (Bullard et al., 2010), which reduces bias in RPKM values caused by highly expressed genes. To identify differentially expressed genes (DEGs) between samples collected at different time-points from four species, we applied edgeR software, based on the $\mathrm{R}$ programming environment (Robinson et al., 2010), with the RPKM values of orthologs between the four poplar species (using 0.001 instead of 0 if the RPKM was 0 , and $\geq 1$ in either of the libraries). In pairwise comparisons using edgeR, the $p$-values for each of the expressed genes were obtained, the false discovery rate (FDR) was used to justify the $p$-value according to the function p.adjust in $\mathrm{R}$. Sequences were deemed to be differentially expressed if $\log _{2}\left(\mathrm{FPKM}_{\mathrm{time} 1} / \mathrm{FPKM}_{\text {time } 2}\right)>1$ or $<-1$, and the adjusted $p$ value (FDR) was $<0.05$ as identified by both metrics. There are 10 possible pairwise comparisons among the five sampling time points for each species.

\subsection{Cluster analysis and functional enrichment}

All DEGs that were identified in all pairwise comparisons were used for cluster analysis and functional enrichment. K-means clustering of transcript expression patterns based on RPKM values, obtained as described above, was performed using the K-Means/KMedians Support Module (KMS) embedded in MultiExperiment Viewer 4.9 (MEV4.9) (http://www.tm4.org/mev.html). Prior to cluster formation, RPKM expression values for each transcript were normalized and then zero-centered by subtracting the mean for each five values for each sampling time point during the salt stress period. The optimal number of clusters was determined using the FOM (Figures of Merit) application (Yeung et al., 2001) within MEV4.9. The K-Means analysis was performed ten times, with each run generating 15 clusters using Euclidean distances. The final output generated 15 clusters in which all the member genes clustered together in at least $80 \%$ of the K-Means runs (Livak and Schmittgen, 2001). The genes in each cluster were then classified according to GO terms, and a Chi-square test was applied to test for enrichment of functional categories with Bonferroni correction (corrected P-value < $0.01)$.

\subsection{Molecular Evolution Analysis}

To evaluate variation in selective pressure in the salt-sensitive species and the salttolerant species, we reconstructed ancestral sequences of the pairs of the orthologs from the two kinds of poplar using the CODEML models in Phylogenetic Analysis by 
Maximum Likelihood (PAML v4.6) (Yang, 2007). We calculated the nonsynonymous $(d N)$ and synonymous $(d S)$ substitution ratios between orthologous ancestral sequences in the PAML using the YN00 model.

\section{Results and Discussion}

3.1. Analysis and mapping of Illumina-Solexa sequencing tags

In order to test for divergence in adaptive gene expression in the ancestral clade of the two salt-tolerant ( $P$. pruinosa and $P$. euphratica) poplars from the pattern of expression in the salt-sensitive poplars, we used the Illumina-Solexa sequencing platform to sequence transcriptomes from salt-stressed calli (exposed to 200mM NaCl for 6, 12, 24, and 48h) and controls for P. pruinosa (Zhang et al., 2014b), P. trichocarpa (in this study), P. euphratica and P. tomentosa (Ma et al., 2013). After removing low-quality sequences and trimming adapter sequences, $\sim 50$ million 100bp paired-end clean reads were generated from each of the cDNA libraries in the Illumina Genome Analyzer runs (Table 1). The expressed reads from each of the four poplar species under salt stress were mapped to the corresponding predicted protein coding genes using Bowtie 2 with default parameters. Approximately $60 \%$ of the reads had matches (Table 1). Additionally, the numbers of mapped genes in each library amounted to $\sim 80 \%$, indicating that most genes were expressed in our salt-stressed calli (Table 1). Among these genes, more than $70 \%$ had RPKM values in the range 1 to 100 for each time point (Fig. S1).

Table 1. Statistics of reads mapped to CDS for the four Populus species

\begin{tabular}{llllll}
\hline Sample ID & & $\begin{array}{l}\text { No. cleaned } \\
\text { reads }\end{array}$ & $\begin{array}{l}\text { No. mapped } \\
\text { reads }\end{array}$ & $\begin{array}{l}\text { Percentage } \\
(\%)\end{array}$ & $\begin{array}{l}\text { Matched } \\
\text { CDS }\end{array}$ \\
\hline P. euphratica & $0 \mathrm{~h}$ & $55,112,298$ & $36,195,071$ & 65.7 & $28,768(84 \%)$ \\
& $6 \mathrm{~h}$ & $48,054,722$ & $32,383,852$ & 67.4 & $27,261(80 \%)$ \\
& $12 \mathrm{~h}$ & $48,199,694$ & $33,361,231$ & 69.2 & $26,875(78 \%)$ \\
& $24 \mathrm{~h}$ & $72,270,670$ & $48,535,155$ & 67.1 & $28,723(84 \%)$ \\
& $48 \mathrm{~h}$ & $48,029,168$ & $33,896,950$ & 70.6 & $26,565(77 \%)$ \\
& $0 \mathrm{~h}$ & $50,025,426$ & $33,394,001$ & 66.8 & $29,129(84 \%)$ \\
& $6 \mathrm{~h}$ & $51,284,884$ & $33,958,235$ & 66.2 & $26,942(78 \%)$ \\
& $12 \mathrm{~h}$ & $53,640,902$ & $35,363,231$ & 65.9 & $26,706(77 \%)$ \\
& $24 \mathrm{~h}$ & $49,571,046$ & $31,995,742$ & 64.6 & $26,472(76 \%)$
\end{tabular}




\begin{tabular}{llllll} 
P. trichocarpa & $0 \mathrm{~h}$ & $67,775,862$ & $46,079,672$ & 68.0 & $31,170(82 \%)$ \\
& $6 \mathrm{~h}$ & $61,413,448$ & $39,461,825$ & 64.3 & $33,614(88 \%)$ \\
\hline & $12 \mathrm{~h}$ & $47,818,814$ & $29,537,544$ & 61.8 & $33,129(87 \%)$ \\
P. tomentosa & $24 \mathrm{~h}$ & $56,590,512$ & $33,164,077$ & 58.6 & $32,797(86 \%)$ \\
& $48 \mathrm{~h}$ & $46,223,672$ & $30,153,003$ & 65.2 & $30,941(81 \%)$ \\
& $6 \mathrm{~h}$ & $59,729,084$ & $36,122,492$ & 60.5 & $32,795(96 \%)$ \\
& $12 \mathrm{~h}$ & $56,452,636$ & $33,477,154$ & 59.3 & $33,565(98 \%)$ \\
& $24 \mathrm{~h}$ & $47,950,660$ & $30,087,577$ & 62.8 & $33,142(97 \%)$ \\
& $48 \mathrm{~h}$ & $56,570,710$ & $35,040,845$ & 61.9 & $33,329(97 \%)$ \\
\hline
\end{tabular}

190

We obtained 34,637, 34,279, 37,949 and 34,177 protein coding genes for $P$. pruinosa, $P$. euphratica, $P$. trichocarpa and $P$. tomentosa, respectively (Table S1). In addition, we identified 7,822 1:1 orthologs between the four poplar species (Table S2). To reduce the effects of background transcription, caused by unavoidable errors during sequence and reads mapping, genes were included in our analysis only if their RPKM values were $\geq 1$ in samples collected at more than one time point. Applying this criterion, 7,752 orthologous genes were considered to be expressed and were selected for further analysis.

To determine the divergence in gene expression between the four poplars in response to salt stress in more detail, we performed a Spearman correlation analysis, with bootstrapping, for the 7,752 orthologous genes from P. pruinosa, P. euphratica, $P$. trichocarpa and P. tomentosa for each time point (Fig. 1). We found that P. pruinosa and $P$. euphratica were clustered together, while $P$. trichocarpa and $P$. tomentosa were also clustered together (Fig. 1). The results of the correlation tests suggested that the patterns of gene expression were significantly different in the two salt-tolerant species ( $P$. pruinosa and $P$. euphratica) compared to those in the two salt-sensitive ones $(P$. trichocarpa and $P$. tomentosa). This suggests that the adaptive patterns of gene expression shown by these two sister desert poplars may have evolved as their ancestral clade diverged from other poplars. Additionally, we found that the control calli from $P$. pruinosa and $P$. euphratica were clustered together, while the salt-stressed calli from $P$. pruinosa and $P$. euphratica were separated. This suggests that these two sister species may have evolved divergent regulatory and metabolic pathways during interaction with 
different salt habitats. The results were in accordance with those reported in our previous studies (Ma et al., 2013; Zhang et al., 2013; Zhang et al., 2014a).

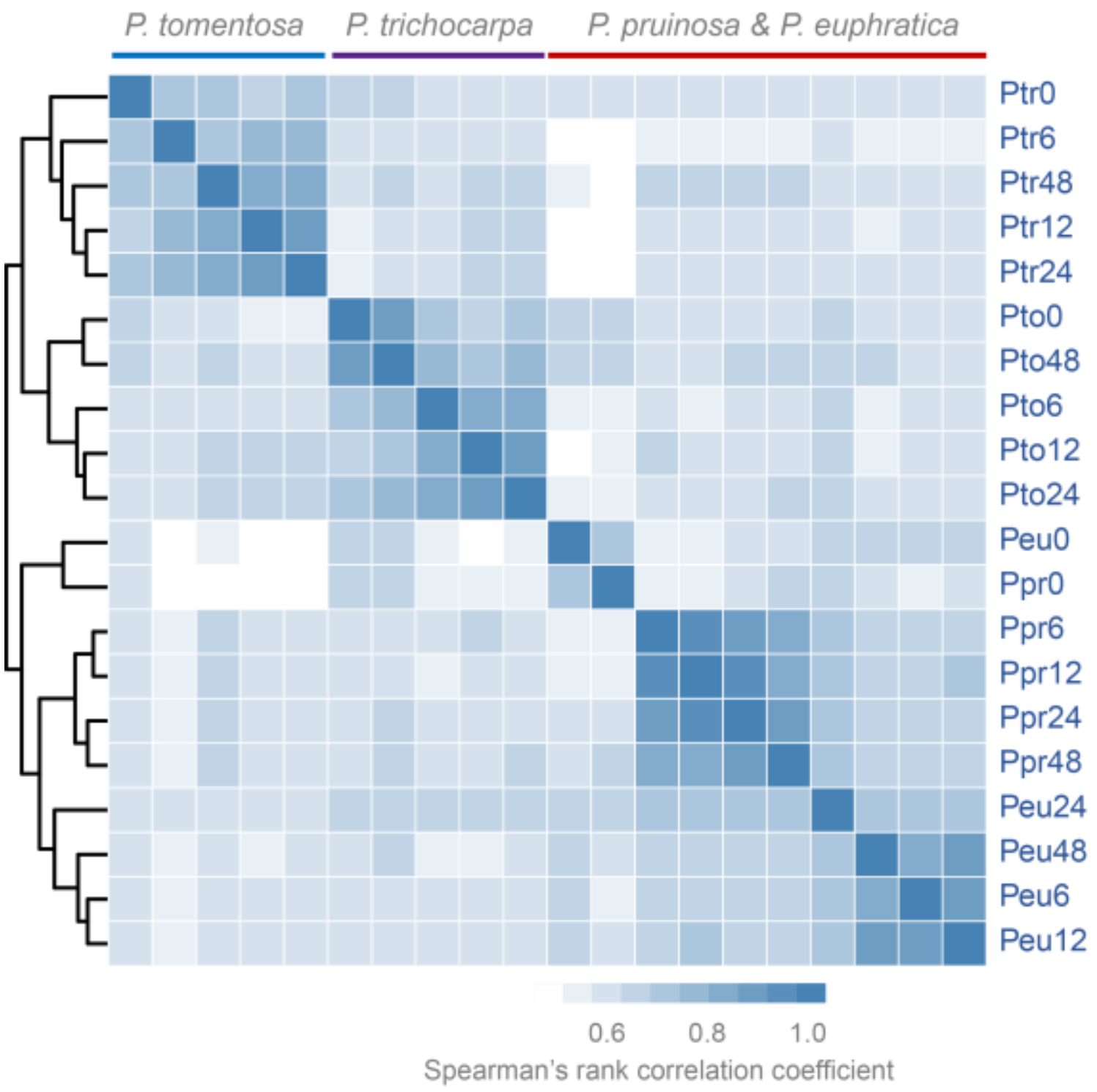

Fig. 1. Hierarchical clustering of all samples by Spearman correlations based on RPKM 217 of 7,752 orthologous genes.

218 3.2. Differentially expressed genes (DEGs) in response to salt stress

219 To identify global transcriptional changes occurring under salt stress, we used edgeR to identify genes that were differentially expressed between samples collected at any pair of time points in each species. We selected those DEGs whose expression profiles met three criteria: (I) the FPKM value was $\geq 1$ in either of the libraries, (II) $\log _{2}$ $\left(\mathrm{FPKM}_{\text {time1 }} / \mathrm{FPKM}_{\text {time2 }}\right)$ was $>1$ or $<-1$, and (III) the adjusted p-value (FDR) was $<0.05$.

224 In total, we identified 1906, 1461, 1776 and 2400 DEGs showing either up- or downregulation in $P$. pruinosa, $P$. euphratica, $P$. trichocarpa and $P$. tomentosa, respectively, at any pair of time points (Fig. S2a and Fig. S2b). On the basis of the 7,822 1:1 orthologs 
between the four poplar species, a total of 1335 differentially expressed orthologous genes were identified. Moreover, the differentially expressed orthologous genes were included in our analysis only if their RPKM values were $>0$ at more than one time point for any species. Applying this criterion, 702 differentially expressed orthologous genes were selected for further analysis. Numbers of unique and shared DEGs between samples of calli exposed to salt stress for $0,6,12,24$ and 48 h are shown in Fig. S2c. Among these DEGs, 109 were expressed in both salt-tolerant species, 287 were specifically expressed in $P$. pruinosa and 159 were specifically expressed in $P$. euphratica during salt stress, while 73 were differentially regulated in salt-sensitive species; 378 and 46 were specifically expressed in P. tomentosa and P. trichocarpa, respectively (Fig. S2c).

\subsection{Identification of temporal expression trends in response to salt stress}

To examine further the temporal trends in expression in calli cultivated from the four species in response to salinity stress, we ran a K-Means clustering algorithm to group the 702 DEGs that were expressed at least once; this yielded fifteen clusters (designated K1-15) of genes with similar patterns of temporal expression (Fig. S3). Genes in clusters $\mathrm{K} 1$ and $\mathrm{K} 13$, which were highly expressed in all four poplar species during almost continuous salinity stress, may be housekeeping genes. In contrast, genes in clusters K8 and K5 were down-regulated in P. trichocarpa and up-regulated in the two salt-tolerant species and $P$. tomentosa, while $\mathrm{K} 10$ and $\mathrm{K} 11$ were down-regulated in $P$. pruinosa and up-regulated in the two salt-sensitive species and P. euphratica. Genes in clusters K2, K6, K12 and K14 were least strongly up-regulated in one of the saltsensitive species and down-regulated in one of the two salt-tolerant species. However, the expression level of genes in clusters $\mathrm{K} 4$ and $\mathrm{K} 7$ changed more rapidly and markedly in the two salt-tolerant species or the two salt-sensitive species. Those that we considered important are listed in Table S2. The candidate genes identified in the present study contained both the previously reported salt-responsive genes and some with species-specific expression. In future studies, these genes of two clusters could be used for allele mining to determine the basis of the common response to stress conditions shown by the two desert poplar species. This clustering analysis allows us to aggregate genes over multiple time points, providing another view of functional transitions of poplar species in response to salinity stress.

3.4. Functional classification of DEGs in response to salt stress 
260 We further analyzed the overrepresented GO functions within each cluster (Fig. 2).

261 Based on the GO enrichment analysis, the biological processes represented are shown in 262 Fig 4. The K3 and K5 clusters contained the most over-represented GO terms among all 263 of the clusters. In K3, and genes involving "Transferase activity", "Transmembrane 264 transporter activity", "Membrane", "Transmembrane transport" were over-represented. 265 In K5, "Photosynthesis", "Thylakoid part", "Membrane protein complex", "Catalytic 266 activity", and "Membrane part" were particularly common. The K2 cluster had high 267 representation of genes involved in "Hydrolase activity", "Binding", "Proteolysis" and 268 "Peptidase activity"; the K10 cluster showed over-representation of "Biological process", "Carbohydrate metabolic process" and "Hydrolase activity". 61 genes 270 involved in "Primary metabolic process", "Organic substance metabolic process", 271 "Sequence-specific DNA binding transcription factor activity" and "Nucleic acid binding transcription factor activity" were greatly enriched in cluster $\mathrm{K} 4$, representing genes consistently up-regulated in the two salt-sensitive species and down-regulated in the two salt-tolerant species, suggesting that the two types of poplars established different energetic and developmental equilibria under salt stress (Bartels and Sunkar, 2005; Ottow et al., 2005). In addition, 36 genes in cluster K7 involved in “Oxidoreductase activity" were greatly over-represented, being continuously upregulated in the two salt-tolerant and down-regulated in the two sensitive species when exposed to salt stress. Genes involved in "Localization", "Establishment of localization" and "Transport" were over-represented in clusters K1, K8, K14 and K15. On the whole, the functional bias of these genes reflects the highly dynamic and coordinated transition of gene expression in the four Populus species' responses to a saline environment. 
Photosynthesis

Thylakoid part

Localization

Establishment of localization $\quad 15$

Transport

Membrane protein complex

Catalytic activity

Protein complex

Intracellular part

Macromolecular complex

Membrane part

Oxidoreductase activity

Biological_process

Carbohydrate metabolic process

Hydrolase activity, acting on glycosyl bonds

Hydrolase activity, hydrolyzing O-glycosyl compounds

Substrate-specific transporter activity

Transmembrane transporter activity

Transporter activity

Transferase activity, transferring hexosyl groups

Membrane

Transferase activity, transferring glycosyl groups

Substrate-specific transmembrane transporter activity

DNA binding

Transmembrane transport

Hydrolase activity, acting on ester bonds

Binding

Proteolysis

Hydrolase activity

Peptidase activity, acting on L-amino acid peptides

Peptidase activity

Biological regulation

Primary metabolic process

Organic substance metabolic process

Sequence-specific DNA binding transcription factor activity

Nucleic acid binding transcription factor activity

Fig. 2. GO-term function enrichment analysis of the 15 clusters. The significances of the most represented GO-slims in each main cluster are indicated using log-transformed $P$ values (red). The gray areas represent missing values.

3.5. Candidate genes of the four Populus species' responses to salt stress

Genes whose expression was highly correlated with salt stress were potential candidates for involvement in responding to salty environments. Based on our analysis, we suggested eleven candidate genes as being continuously expressed differently in both $P$. pruinosa and $P$. euphratica as compared to $P$. trichocarpa and $P$. tomentosa with respect to responses to salt stress and found in clusters K4 and K7 (Fig. 3). In cluster K4, we identified numerous genes with significant up-regulation in both the two salt-sensitive species when stressed, including 6-phosphogluconolactonase 4, Raffinose synthase, UDP-glucuronosyl transferase, Heat shock protein Hsp2O and Hsp70, Zinc finger, WRKY, Peroxidase 15, Lipase GDSL, Paratin, ERF, Fatty acid desaturase, bZIP and Protein kinase (Fig. 3a). All these identified DEGs would have also played an important role in salt-sensitive species during salt adaptation. Their functions and molecular 
299 mechanisms need further clarification in the future to examine enhancing salt stress 300 tolerance in salt-sensitive crops. In cluster K7, we found six genes that were enriched 301 with respect to "Oxidoreductase activity", 2 Plant peroxidase (Meloni et al., 2003; 302 Yoshida et al., 2003; Fujita et al., 2006), Plant disease resistance response protein, 303 NADH ( Sobhanian et al., 2010), cytochrome P450 (Narusaka et al., 2004) and Lipase 304 GDSL (Naranjo et al., 2006); these genes have been found to be related to salt-response 305 in other studies ( $\mathrm{Li}$ et al., 2013). The specific RPKM values of the eleven candidate 306 genes at the five time points in the two types of poplar are shown in Fig. 3b. The 307 expression of cytochrome $P 450$, which is related to salt stress, was enhanced in the salt308 sensitive poplars, but decreased in the two salt-tolerant species when exposed to 309 continuous salinity stress. This indicated that cytochrome $P 450$ played an important role 310 in the process of responding to salt stress in the different poplars. 
a
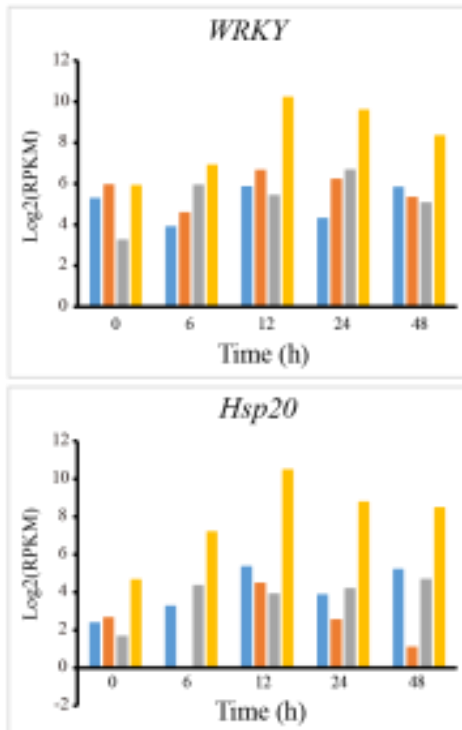

b

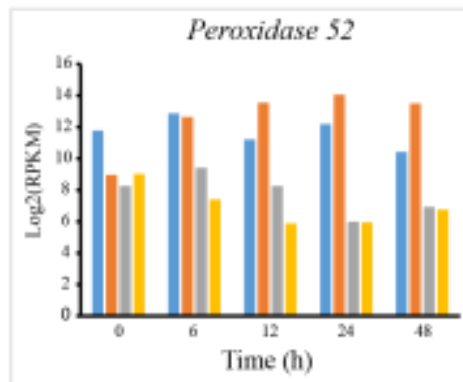

GDSL esterase/lipase I

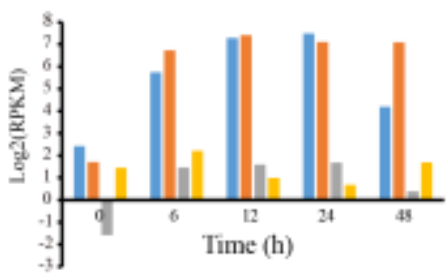

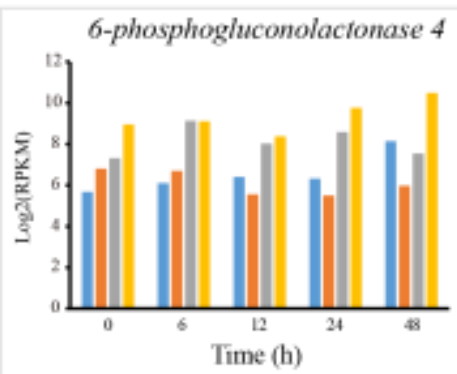
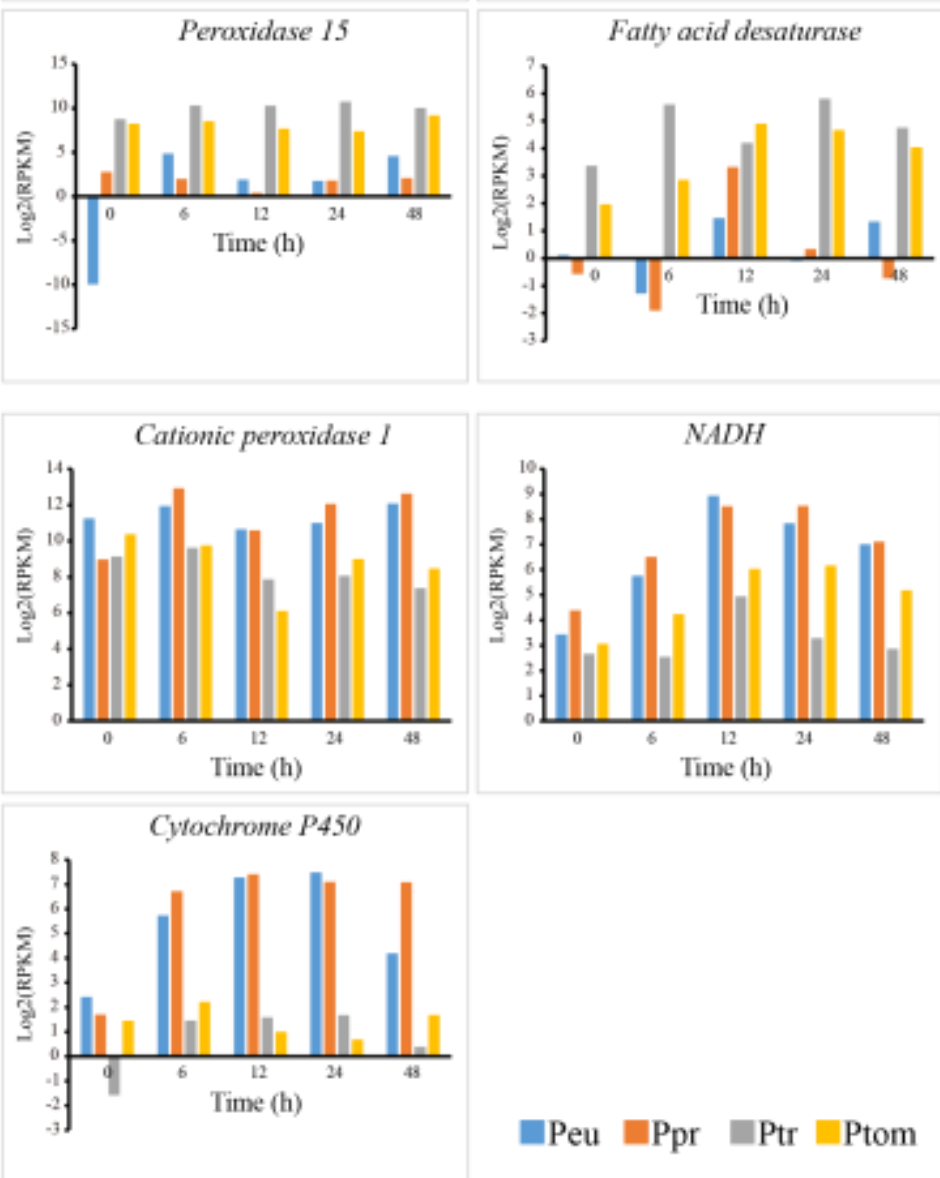

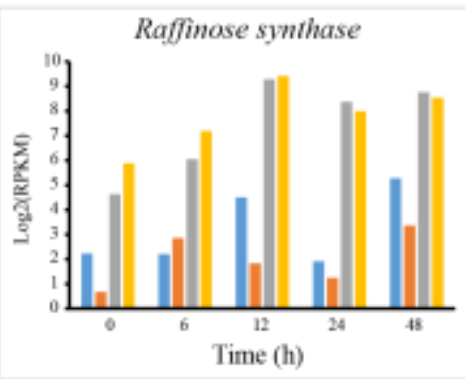

Fatty acid desaturase

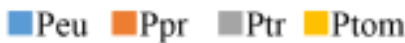

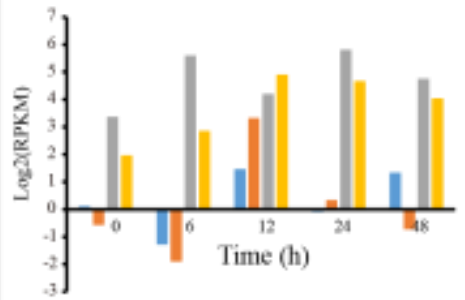

Fig. 3. The RPKM values of 11 candidate genes related to the salt response in two types of poplar at five time points. The expression level of five candidate genes of cluster K4 (a) and six candidate genes of cluster K7 (b). The y-axis shows the log2-transformed RPKM values of genes expressed after different periods of salt stress, and the $\mathrm{x}$-axis shows the duration of salt stress.

3.6. Molecular Evolution of the Candidate genes of the four Populus species

To determine the influence of selection on the pairs of orthologs from the two saltsensitive species and the two salt-tolerant species, the ratio of non-synonymous vs. synonymous substitutions $(\omega=d N / d S)$ was used because it is an indicator of the history of selection acting on a gene (Table S3). Ratios significantly $<1$ are suggestive of purifying selection which hinders the spread of deleterious alleles, whereas ratios $>1$ 
suggest positive selection which promotes the spread of beneficial alleles in a population. We reconstructed ancestral sequences of the pairs of the orthologs from the salt-sensitive species and the salt-tolerant species, respectively. A plot of $d N / d S$ for the orthologous candidate genes is shown in Figure S4 and the results suggest that most pairs evolved mainly under the influence of purifying selection. Of these, one pair of orthologs $(G D S L)$ between the two types of poplar has a $d N / d S>1$, indicating positive selection, and five pairs have a $d N / d S$ between 0.5 and 1, indicating weak purifying selection. Our findings together indicated that the two types of poplars may have developed species-specific regulatory mechanisms for adaptation to salinity during their ecological speciation.

\section{Acknowledgements:}

\section{References}

Alexeyenko, A., Tamas, I., Liu, G. and Sonnhammer, E.L., 2006. Automatic clustering of orthologs and inparalogs shared by multiple proteomes. Bioinformatics 22, e9-e15.

Álvarez, S. and Sánchez - Blanco, M., 2014. Long - term effect of salinity on plant quality, water relations, photosynthetic parameters and ion distribution in Callistemon citrinus. Plant Biology 16, 757-764.

Bartels, D. and Sunkar, R., 2005. Drought and salt tolerance in plants. Crit. Rev. Plant Sci. 24, 23-58.

Bazihizina, N., Barrett-Lennard, E.G. and Colmer, T.D., 2012. Plant growth and physiology under heterogeneous salinity. Plant Soil 354, 1-19.

Beritognolo, I., Harfouche, A., Brilli, F., Prosperini, G., Gaudet, M., Brosché, M., Salani, F., Kuzminsky, E., Auvinen, P. and Paulin, L., 2011. Comparative study of transcriptional and physiological responses to salinity stress in two contrasting Populus alba L. genotypes. Tree Physiol., tpr083.

Blanchette, M., Kent, W.J., Riemer, C., Elnitski, L., Smit, A.F., Roskin, K.M., Baertsch, R., Rosenbloom, K., Clawson, H. and Green, E.D., 2004. Aligning multiple genomic sequences with the threaded blockset aligner. Genome Res. 14, 708715.

Bullard, J.H., Purdom, E., Hansen, K.D. and Dudoit, S., 2010. Evaluation of statistical methods for normalization and differential expression in mRNA-Seq experiments. BMC Bioinformatics 11, 94.

Cabot, C., Sibole, J.V., Barceló, J. and Poschenrieder, C., 2014. Lessons from crop plants struggling with salinity. Plant Sci. 226, 2-13.

Cohen, D., Bogeat-Triboulot, M.-B., Tisserant, E., Balzergue, S., Martin-Magniette, M.L., Lelandais, G., Ningre, N., Renou, J.-P., Tamby, J.-P. and Le Thiec, D., 2010. Comparative transcriptomics of drought responses in Populus: a meta-analysis of genome-wide expression profiling in mature leaves and root apices across two genotypes. BMC Genomics 11, 630.

Cushman, J.C. and Bohnert, H.J., 2000. Genomic approaches to plant stress tolerance. 
Curr. Opin. Plant Biol. 3, 117-124.

Deyholos, M.K., 2010. Making the most of drought and salinity transcriptomics. Plant Cell Environ. 33, 648-654.

Gharat, S.A., Parmar, S., Tambat, S., Vasudevan, M. and Shaw, B.P., 2016. Transcriptome Analysis of the Response to $\mathrm{NaCl}$ in Suaeda maritima Provides an Insight into Salt Tolerance Mechanisms in Halophytes. PLoS One 11, $\mathrm{e} 0163485$.

Gong, Q., Li, P., Ma, S., Indu Rupassara, S. and Bohnert, H.J., 2005. Salinity stress adaptation competence in the extremophile Thellungiella halophila in comparison with its relative Arabidopsis thaliana. Plant J. 44, 826-839.

Ingram, J. and Bartels, D., 1996. The molecular basis of dehydration tolerance in plants. Annu. Rev. Plant Biol. 47, 377-403.

Jiang, S.-Y., Ma, A., Ramamoorthy, R. and Ramachandran, S., 2013. Genome-wide survey on genomic variation, expression divergence, and evolution in two contrasting rice genotypes under high salinity stress. Genome Bio. Evol. 5, 2032-2050.

Langmead, B., Trapnell, C., Pop, M. and Salzberg, S.L., 2009. Ultrafast and memoryefficient alignment of short DNA sequences to the human genome. Genome Biol. $10, \mathrm{R} 25$.

Lasky, J.R., Des Marais, D.L., Lowry, D.B., Povolotskaya, I., McKay, J.K., Richards, J.H., Keitt, T.H. and Juenger, T.E., 2014. Natural variation in abiotic stress responsive gene expression and local adaptation to climate in Arabidopsis thaliana. Mol. Biol. Evol. 31, 2283-2296.

Li, B., Duan, H., Li, J., Deng, X.W., Yin, W. and Xia, X., 2013. Global identification of miRNAs and targets in Populus euphratica under salt stress. Plant Mol. Biol. 81, 525-539.

Liu, C.-h., Lu, R.-j., Guo, G.-m., He, T., Li, Y.-b., Xu, H.-w., Gao, R.-h., Chen, Z.-w. and Huang, J.-h., 2016. Transcriptome analysis reveals translational regulation in barley microspore-derived embryogenic callus under salt stress. Plant Cell Rep., 1-10.

Livak, K.J. and Schmittgen, T.D., 2001. Analysis of relative gene expression data using real-time quantitative PCR and the 2(-Delta Delta C(T)) method. Methods 25, 402-408.

Ma, T., Wang, J., Zhou, G., Yue, Z., Hu, Q., Chen, Y., Liu, B., Qiu, Q., Wang, Z. and Zhang, J., 2013. Genomic insights into salt adaptation in a desert poplar. Nat. Commun. 4.

Mortazavi, A., Williams, B.A., McCue, K., Schaeffer, L. and Wold, B., 2008. Mapping and quantifying mammalian transcriptomes by RNA-Seq. Nat. Methods 5, 621628.

Munns, R. and Tester, M., 2008. Mechanisms of salinity tolerance. Annu. Rev. Plant Biol. 59, 651-681.

O'Neil, S.T., Dzurisin, J.D., Williams, C.M., Lobo, N.F., Higgins, J.K., Deines, J.M., Carmichael, R.D., Zeng, E., Tan, J.C. and Wu, G.C., 2014. Gene expression in closely related species mirrors local adaptation: consequences for responses to a warming world. Mol. Ecol. 23, 2686-2698.

Östlund, G., Schmitt, T., Forslund, K., Köstler, T., Messina, D.N., Roopra, S., Frings, O. and Sonnhammer, E.L., 2010. InParanoid 7: new algorithms and tools for eukaryotic orthology analysis. Nucleic Acids Res. 38, D196-D203.

Ottow, E.A., Brinker, M., Teichmann, T., Fritz, E., Kaiser, W., Brosché, M., Kangasjärvi, J., Jiang, X. and Polle, A., 2005. Populus euphratica displays apoplastic sodium 
accumulation, osmotic adjustment by decreases in calcium and soluble carbohydrates, and develops leaf succulence under salt stress. Plant Physiol. 139, 1762-1772.

Qiu, Q., Ma, T., Hu, Q., Liu, B., Wu, Y., Zhou, H., Wang, Q., Wang, J. and Liu, J., 2011. Genome-scale transcriptome analysis of the desert poplar, Populus euphratica. Tree Physiol., tpr015.

Rengasamy, P., 2006. World salinization with emphasis on Australia. J. Exp. Bot. 57, $1017-23$.

Robinson, M.D., McCarthy, D.J. and Smyth, G.K., 2010. edgeR: a Bioconductor package for differential expression analysis of digital gene expression data. Bioinformatics 26, 139-140.

Sahu, B.B. and Shaw, B.P., 2009. Isolation, identification and expression analysis of salt-induced genes in Suaeda maritima, a natural halophyte, using PCR-based suppression subtractive hybridization. BMC Plant Biol. 9, 1.

Shinozaki, K., Yamaguchi-Shinozaki, K. and Seki, M., 2003. Regulatory network of gene expression in the drought and cold stress responses. Curr. Opin. Plant Biol. 6, 410-417.

Sun, X., Xu, L., Wang, Y., Luo, X., Zhu, X., Kinuthia, K.B., Nie, S., Feng, H., Li, C. and Liu, L., 2016. Transcriptome-based gene expression profiling identifies differentially expressed genes critical for salt stress response in radish (Raphanus sativus L.). Plant Cell Rep. 35, 329-346.

Taji, T., Seki, M., Satou, M., Sakurai, T., Kobayashi, M., Ishiyama, K., Narusaka, Y., Narusaka, M., Zhu, J.-K. and Shinozaki, K., 2004. Comparative genomics in salt tolerance between Arabidopsis and Arabidopsis-related halophyte salt cress using Arabidopsis microarray. Plant Physiol. 135, 1697-1709.

Tang, X., Wang, H., Shao, C. and Shao, H., 2015. Global gene expression of Kosteletzkya virginica seedlings responding to salt stress. PLoS One 10, e0124421.

Tuskan, G.A., Difazio, S., Jansson, S., Bohlmann, J., Grigoriev, I., Hellsten, U., Putnam, N., Ralph, S., Rombauts, S. and Salamov, A., 2006. The genome of black cottonwood, Populus trichocarpa (Torr. \& Gray). Science 313, 1596-1604.

Tuteja, N., 2007. Mechanisms of high salinity tolerance in plants. Method. Enzymol. 428, 419-38.

Witzel, K., Weidner, A., Surabhi, G.-K., Börner, A. and Mock, H.-P., 2009. Salt stressinduced alterations in the root proteome of barley genotypes with contrasting response towards salinity. J. Exp. Bot. 60, 3545-3557.

Wong, C.E., Li, Y., Labbe, A., Guevara, D., Nuin, P., Whitty, B., Diaz, C., Golding, G.B., Gray, G.R. and Weretilnyk, E.A., 2006. Transcriptional profiling implicates novel interactions between abiotic stress and hormonal responses in Thellungiella, a close relative of Arabidopsis. Plant Physiol. 140, 1437-1450.

Yang, Z., 2007. PAML 4: phylogenetic analysis by maximum likelihood. Mol. Biol. Evol. 24, 1586-91.

Yeo, A., 1998. Predicting the interaction between the effects of salinity and climate change on crop plants. Sci. Hortic-Amsterdam 78, 159-174.

Yeung, K.Y., Haynor, D.R. and Ruzzo, W.L., 2001. Validating clustering for gene expression data. Bioinformatics 17, 309-318.

Zhang, J.-L. and Shi, H., 2013. Physiological and molecular mechanisms of plant salt tolerance. Photosynth. Res. 115, 1-22.

Zhang, J., Feng, J., Lu, J., Yang, Y., Zhang, X., Wan, D. and Liu, J., 2014a. Transcriptome differences between two sister desert poplar species under salt 
stress. BMC Genomics 15, 337.

Zhang, J., Jiang, D., Liu, B., Luo, W., Lu, J., Ma, T. and Wan, D., 2014b. Transcriptome dynamics of a desert poplar (Populus pruinosa) in response to continuous salinity stress. Plant Cell Rep. 33, 1565-1579.

Zhang, J., Xie, P., Lascoux, M., Meagher, T.R. and Liu, J., 2013. Rapidly evolving genes and stress adaptation of two desert poplars, Populus euphratica and $P$. pruinosa. PLoS One 8, e66370.

Zhu, J.-K., 2002. Salt and drought stress signal transduction in plants. Annu. Rev. Plant Biol. 53, 247. 
Table 1. Statistics of reads mapped to CDS for the four Populus species

\begin{tabular}{|c|c|c|c|c|c|}
\hline Sample ID & & $\begin{array}{l}\text { No. cleaned } \\
\text { reads }\end{array}$ & $\begin{array}{l}\text { No. mapped } \\
\text { reads }\end{array}$ & $\begin{array}{l}\text { Percentage } \\
(\%)\end{array}$ & $\begin{array}{l}\text { Matched } \\
\text { CDS }\end{array}$ \\
\hline \multirow[t]{5}{*}{ P. euphratica } & $0 \mathrm{~h}$ & $55,112,298$ & $36,195,071$ & 65.68 & $28,768(84 \%)$ \\
\hline & $6 \mathrm{~h}$ & $48,054,722$ & $32,383,852$ & 67.39 & $27,261(80 \%)$ \\
\hline & $12 \mathrm{~h}$ & $48,199,694$ & $33,361,231$ & 69.21 & $26,875(78 \%)$ \\
\hline & $24 \mathrm{~h}$ & $72,270,670$ & $48,535,155$ & 67.12 & $28,723(84 \%)$ \\
\hline & $48 \mathrm{~h}$ & $48,029,168$ & $33,896,950$ & 70.58 & $26,565(77 \%)$ \\
\hline \multirow[t]{5}{*}{ P. pruinosa } & $0 \mathrm{~h}$ & $50,025,426$ & $33,394,001$ & 66.75 & $29,129(84 \%)$ \\
\hline & $6 \mathrm{~h}$ & $51,284,884$ & $33,958,235$ & 66.21 & $26,942(78 \%)$ \\
\hline & $12 \mathrm{~h}$ & $53,640,902$ & $35,363,231$ & 65.93 & $26,706(77 \%)$ \\
\hline & $24 \mathrm{~h}$ & $49,571,046$ & $31,995,742$ & 64.55 & $26,472(76 \%)$ \\
\hline & $48 \mathrm{~h}$ & $46,246,696$ & $29,712,865$ & 64.25 & $26,724(77 \%)$ \\
\hline \multirow[t]{5}{*}{ P. trichocarpa } & $0 \mathrm{~h}$ & $67,775,862$ & $46,079,672$ & 67.99 & $31,170(82 \%)$ \\
\hline & $6 \mathrm{~h}$ & $61,413,448$ & $39,461,825$ & 64.26 & $33,614(88 \%)$ \\
\hline & $12 \mathrm{~h}$ & $47,818,814$ & $29,537,544$ & 61.77 & $33,129(87 \%)$ \\
\hline & $24 \mathrm{~h}$ & $56,590,512$ & $33,164,077$ & 58.60 & $32,797(86 \%)$ \\
\hline & $48 \mathrm{~h}$ & $46,223,672$ & $30,153,003$ & 65.23 & $30,941(81 \%)$ \\
\hline \multirow[t]{5}{*}{ P. tomentosa } & $0 \mathrm{~h}$ & $59,729,084$ & $36,122,492$ & 60.48 & $32,795(96 \%)$ \\
\hline & $6 \mathrm{~h}$ & $56,452,636$ & $33,477,154$ & 59.30 & $33,565(98 \%)$ \\
\hline & $12 \mathrm{~h}$ & $55,423,950$ & $34,443,117$ & 62.14 & $33,142(97 \%)$ \\
\hline & $24 \mathrm{~h}$ & $47,950,660$ & $30,087,577$ & 62.75 & $33,329(97 \%)$ \\
\hline & $48 \mathrm{~h}$ & $56,570,710$ & $35,040,845$ & 61.94 & $33,470(98 \%)$ \\
\hline
\end{tabular}

